\title{
Hormonal causes of recurrent pregnancy loss (RPL)
}

\author{
Nicola Pluchino, ${ }^{1}$ Panagiotis Drakopoulos, ${ }^{1}$ Jean Marie Wenger, ${ }^{1}$ Patrick Petignat, ${ }^{1}$ \\ Isabelle Streuli, ${ }^{1}$ Andrea Riccardo Genazzani ${ }^{2}$
}

${ }^{1}$ Division of Obstetrics and Gynaecology, University Hospitals of Geneva, Switzerland; ${ }^{2}$ Division of Obstetrics and Gynaecology, University Hospitals of Pisa, Italy

\begin{abstract}
Endocrine disorders play a major role in approximately $8 \%$ to $12 \%$ of recurrent pregnancy loss (RPL). Indeed, the local hormonal milieu is crucial in both embryo attachment and early pregnancy. Endocrine abnormalities, including thyroid disorders, luteal phase defects, polycystic ovary syndrome, hyperprolactinaemia and diabetes have to be evaluated in any case of RPL. Moreover, elevated androgen levels and some endocrinological aspects of endometriosis are also factors contributing to RPL. In the present article, we review the significance of endocrine disease on RPL.
\end{abstract}

Key words: Abortion, Hyperprolactinaemia, Luteal phase defect, Miscarriage, Recurrent pregnancy loss

\section{INTRODUCTION}

The development and the maintenance of pregnancy are dependent on numerous endocrinological events that lead to the successful growth and development of the foetus. Although the vast majority of pregnant women have no pre-existing endocrine abnormalities, a small percentage of women may develop endocrine alterations that could potentially lead to sporadic or recurrent miscarriage. It is estimated that approximately $8 \%$ to $12 \%$ of all cases of recurrent pregnancy loss (RPL) are caused by endocrine diseases. ${ }^{1}$ Disorders related to inadequate progesterone secretion by the corpus luteum, hyperprolactinaemia, diabetes

\footnotetext{
Address for correspondence:

Panagiotis Drakopoulos, Boulevard de la Cluse 30,

1205, Geneva, Switzerland, Tel.: +30 795532356

E-mail: Panagiotis.Drakopoulos@hcuge.ch

Received: 01-05-2014, Accepted: 08-05-2014
}

mellitus and polycystic ovarian syndrome are some examples of endocrine disorders affecting pregnancy. Meanwhile, hyperinsulinaemia and elevated androgen levels have recently been added to the endocrinological abnormalities associated with RPLs.

There are two types of miscarriages: sporadic and recurrent. Approximately 30\% to 50\% of all conceptions and $15 \%$ of clinically recognized pregnancies ( $\geq 6$ weeks of gestation) fail to result in a live birth, this due to random foetal chromosomal abnormality in $50 \%-60 \%$ of cases. ${ }^{2}$ RPL comes about through a number of causes (genetic, anatomical, hormonal, infections, etc.) and is defined by two or more clinically failed pregnancies detected via ultrasonography or histopathologic examination. ${ }^{3}$ However, as it lacks a consistent definition, some clinicians continue to define RPL as consisting of three or more consecutive pregnancy losses. Indeed, requiring three failed 
pregnancies prior to initiating work-up may offer little additional clinical insight compared to testing after the second loss. Accurate prevalence is not available, but it has been estimated that $2 \%-5 \%$ of women experience RPL with the majority of these cases occurring before 10 weeks of gestation. ${ }^{4}$ The pathophysiological mechanisms of RPL are as yet poorly understood and the aetiologies remain unexplained in up to $50 \%$ of affected couples. This reproductive disorder is a challenge to the clinician because genetic, endocrinological, anatomic, immunologic, thrombophilic and iatrogenic data may be required for a complete evaluation. Foetal aneuploidy is the most common cause of sporadic miscarriage. However, it should be noted that only $4 \%$ of couples with RPL have one partner carrying a balanced translocation, including reciprocal and Robertsonian translocations, resulting in unbalanced translocation in the foetus. ${ }^{5}$

The purpose of this article is to review the pathophysiology of endocrinological diseases causing RPL.

\section{LUTEAL PHASE DEFICIENCY}

Progesterone production triggers morphological and physiological changes in the endometrium creating a suitable environment for the embryo during the implantation window [5-10 days after the luteinizing hormone (LH) surge]. In addition, progesterone helps in maintaining early pregnancy. More specifically, progesterone affects the proliferation and differentiation of stromal cells and augments uterine receptivity through the modulation of locally acting growth factors and regulation of cytokine production in the maternal-foetal interface. Indeed, human and animal studies suggest that progesterone maintains pregnancy by down-regulation of Th1 cytokines and stimulation of Th2 cytokines. ${ }^{6}$ There is evidence that Th2 cytokines favour normal pregnancy, while an excess of Th1 cytokines leads to pregnancy termination. ${ }^{7}$ In particular, in the presence of progesterone, the lymphocytes of pregnant women release a 35 $\mathrm{kD}$ protein named the progesterone-induced blocking factor (PIBF), which in turn alters the profile of cytokine secretion of activated lymphocytes shifting the balance towards Th2 dominance.

All these changes fail to ensue if progesterone production is lower than the normal minimum. In early pregnancy, the corpus luteum continues to produce progesterone until the luteal placental shift. Luteal phase deficiency (LPD) was originally thought to derive from inadequate production of progesterone by the corpus luteum and subsequent inadequate endometrial maturation to allow proper placentation. Those supporting the presence of LPD have theorized that LPD occurs because of poor follicular development, decreased progesterone production by the corpus luteum and a dysfunctional endometrial response to normal progesterone levels. ${ }^{8}$ However, other hypothesized causes of LPD include stress, exercise, weight loss, hyperprolactinaemia and menstrual cycles at the onset of puberty or perimenopause. ${ }^{9}$ Abnormalities of the luteal phase have been historically reported to occur in up to $35 \%$ of women with RPL. ${ }^{1}$ However, actual presence of such a defect and its relation to miscarriage is a controversial issue and there is no consensus on the best method of diagnosis (such as serum progesterone, endometrial biopsy). Serum progesterone levels greater than $10 \mathrm{ng} / \mathrm{mL}$ in the mid-luteal phase are rarely associated with an abnormal luteal phase. ${ }^{11,12}$ Although serum progesterone levels below $<12 \mathrm{ng} / \mathrm{ml}$ have been associated with an increased risk of miscarriage, ${ }^{13}$ its levels can fluctuate during measurements since its secretion is pulsatile and therefore the interpretation of progesterone levels may be difficult. Consistently low progesterone levels may indicate insufficient progesterone to maintain pregnancy; alternatively, it may be the result of the failing pregnancy which produces low hCG leading to low progesterone levels. In the latter case, low progesterone levels seem to be the mechanism leading to expulsion of the conceptus rather than the cause of abortion. Historically, endometrial biopsy, with evaluation of the morphological changes, was considered superior to serum progesterone. However, histological analysis is no longer the gold standard for assessment of endometrial maturation. More recently, immunohistochemical molecular markers (including cytokines and adhesion molecules) have been used for cell biological assessment of endometrial receptivity, while a less traumatic method has been also proposed. ${ }^{14,15}$

Progesterone supplementation after ovulation with or without the use of ovulation-induction agents can also be applied 2 to 3 days after the basal body 
temperature increases (or after a positive urinary LH test) and continued for up to 7 to 11 weeks of gestation. ${ }^{16}$ Progesterone supplementation can be administered by intravaginal suppositories, intramuscular injection of progesterone in oil, as oral micronized progesterone, or as oral dydrogesterone. The subject of progesterone administration continues to be controversial. Daya's meta-analysis demonstrated that hormone treatment to enhance progesterone production or supplementation is associated with an increased chance of a term pregnancy in women with RPL. ${ }^{17}$ However, the more recent Cochrane database systematic review by Oates-Whitehead, ${ }^{18}$ which analyzed the same three papers used in Daya's meta-analysis ten years earlier, found no association with improved pregnancy outcome. Currently, a twocentre trial (PROMISE) is taking place, the results of which are eagerly awaited.

\subsection{Luteal phase defect: lesson learnt from IVF}

Stimulated IVF cycles are associated with a defective luteal phase in almost all patients and progesterone supplementation appears to be mandatory to improve pregnancy rate. ${ }^{19}$ The aetiology of luteal phase defect IVF has been debated for more than two decades and different mechanisms have been proposed. Recently, it has been postulated that one of the main causes of the luteal phase defect in stimulated IVF cycles is related to the supraphysiological levels of steroids secreted by a high number of corpora lutea during the early luteal phase, which directly inhibit LH release via negative feedback actions at the hypothalamic-pituitary axis level. ${ }^{16}$

Studies in humans have demonstrated that the corpus luteum requires a consistent LH stimulus in order to perform its physiological function. LH support during the luteal phase is entirely responsible for the maintenance and the normal steroidogenic activity of the corpus luteum. As a result, withdrawal of LH unnecessarily causes premature luteolysis. ${ }^{20}$

In addition, supraphysiological concentrations of progesterone in stimulated cycles also cause an accelerated transformation to secretory endometrium at the time of embryo transfer, which has been shown to be detrimental to implantation rates. Immunohistochemical studies and microarray technology have indicated an effect of ovarian stimulation on molecular processes involved in implantation that are differentially expressed in stimulated compared to natural cycles. ${ }^{21}$ Understanding and ameliorating the impact of hormones on the natural cycle and during ovarian stimulation on the intrauterine environment may result in improved implantation rates in the future.

\subsection{Hyperprolactinaemia}

Prolactin (PRL) is mainly synthesized and secreted by the lactotroph cells of the pituitary, but also by other sites, such as the mammary gland, placenta, uterus and T lymphocytes. Evidence shows that PRL is essential to female reproduction. PRL is commonly measured in women with RPL, as elevated PRL levels are associated with ovulatory dysfunction. Past in vitro studies have shown that PRL plays a critical role in corpus luteum maintenance and progesterone production in rodents, but not in humans. ${ }^{22}$ Moreover, progesterone secretion by cultured granulosa cells obtained from human ovarian follicles is almost completely inhibited by high PRL concentrations $(100 \mathrm{ng} / \mathrm{mL})$, but not by lower concentrations (10 to $20 \mathrm{ng} / \mathrm{mL}$ ). ${ }^{23}$ These observations suggest the possibility that high PRL concentrations in the early phase of follicular growth may inhibit progesterone secretion, resulting in luteal-phase defects. By contrast, more recent researches on rodents have revealed that PRL receptors are involved not only in generating but also in maintaining pregnancy. However, the precise cellular mechanism of PRL action in the human ovary remains to be clarified. A randomized control trial of 64 hyperprolactinaemic women with RPL treated with bromocriptine was associated with a higher rate of successful pregnancy, and PRL levels were significantly higher in women that miscarried. ${ }^{24}$ Treatment resulted in an $85.7 \%$ live birth rate, whereas the untreated cohort had a $52.4 \%$ live birth outcome. Bromocriptine was given before conception and continued until the end of the 9th week of gestation in the group of patients in whom the serum PRL levels were normalized. On the other hand, in a more recent study of 122 subjects with RPL, only three of them had marginally elevated PRL levels and one a significantly high level (>100 $\mathrm{ng} / \mathrm{ml}) .{ }^{25}$ In conclusion, normal PRL levels may play an important role in the growth and maintenance of early pregnancy, but further studies are required to clarify the role of PRL in the pathogenesis of recur- 
rent miscarriages and to establish whether, in cases of hyperprolactaemia, continuation of treatment during pregnancy may be useful.

\section{THYROID ABNORMALITIES}

\subsection{Hyperthyroidism}

Hyperthyroidism occurs in approximately $0.1 \%$ $0.4 \%$ of pregnancies. ${ }^{26}$ It seems that excess production of thyroid hormone is usually not correlated with infertility or RPL. Pregnant women with untreated excess hyperthyroidism are at increased risk for spontaneous miscarriage, congestive heart failure, thyroid storm, preterm delivery, pre-eclampsia, foetal growth restriction and increased perinatal morbidity and mortality. ${ }^{27}$ Treatment of overt Graves' hyperthyroidism in pregnancy to achieve adequate metabolic control has been associated with improved pregnancy outcomes. However, hyperthyroidism has not commonly been reported as an independent cause of RPL. Only one retrospective study has suggested that excess exogenous thyroid hormone is associated with an elevated rate of foetal loss. ${ }^{28}$

\subsection{Hypothyroidism}

The most prevalent cause of hypothyroidism in pregnant women, affecting approximately $0.5 \%$ of patients, is chronic autoimmune thyroiditis (Hashimoto's thyroiditis).$^{29}$ Other causes of hypothyroidism include endemic iodine deficiency, prior radioactive iodine therapy and thyroidectomy. Untreated hypothyroidism in pregnancy has consistently been shown to be associated with an increased risk for adverse pregnancy complications as well as detrimental effects on foetal neurocognitive development. Specific adverse outcomes associated with maternal overt hypothyroidism include increased risks for premature birth, low birth weight and miscarriage..$^{30}$ Current evidence suggests that treated thyroid dysfunction is not associated with RPL. For this reason, patients should be euthyroid before attempting pregnancy and it is necessary to regularly control the levels of TSH during pregnancy. Thyroid hormones have an impact on oocytes at the level of the granulosa and luteal cells that interfere with normal ovulation. ${ }^{31}$ Low thyroxine levels exert a positive feedback effect on thyroidreleasing hormone (TRH). High concentrations in
TRH have been associated with high PRL levels. It is believed that high PRL levels alter the pulsatility of gonadotropin-releasing hormone $(\mathrm{GnRH})$ and interfere with normal ovulation. Therefore, severe forms of hypothyroidism rarely complicate pregnancy since they are more closely associated with anovulation and infertility. Even if an association exists between low thyroid function and pregnancy loss, there is no direct evidence for a causal role. ${ }^{32}$ We believe it prudent to screen for thyroid disease by measuring TSH levels and normalizing thyroid function prior to conception when the function is found to be abnormal. There is disagreement as to the suitable upper limit of normal serum thyroid-stimulating hormone (TSH) in order to make the diagnosis of subclinical hypothyroidism. The trend regarding the new TSH assays is to decrease the upper limit of normal TSH (range, 4.5 to $5 \mathrm{mU} / \mathrm{L}$ ) to $2.5 \mathrm{mU} / \mathrm{L}$. This upper limit is recommended by the National Academy of Clinical Biochemistry guidelines and is based on the fact that $2.5 \mathrm{mU} / \mathrm{L}$ represents more than two standard deviations above meticulously screened euthyroid volunteers. ${ }^{33}$

\subsection{Thyroid Autoimmunity}

Autoimmune thyroid disease is the most common endocrine disorder in women of reproductive age, with an overall prevalence in women of $10 \%$ to $15 \% .^{34}$ The role of thyroid autoantibodies is debatable with regard to whether there is a causal relationship with RPL. In recent years, studies have found an association between thyroid autoimmunity (TA) and recurrent abortions; moreover, it has been suggested that thyroid autoantibodies may be employed as a marker for at-risk pregnancies. ${ }^{35}$ These studies have linked TA with recurrent miscarriages, although the mechanism involved is not completely understood. Two mechanisms have been postulated to explain the possible association between TA and early pregnancy loss. 1) The presence of TA, which reflects a generalized activation of the immune system and a generally heightened autoimmune reactivity against the foeto-placental unit. ${ }^{36}$ This notion is supported by the observation that women with recurrent abortions have an increased number of CD5/20-positive B cells as compared with women with a normal pregnancy or with only one abortion. ${ }^{37}$ In addition, women with RPL present a significant increase in the endometrial Th1 
cell population that is associated with hypersecretion of INFg and reduced secretion of IL-4 and IL-10. ${ }^{38}$ Dominant pro-inflammatory $\mathrm{Th} 1 \mathrm{immune}$ responses are related to recurrent spontaneous abortions. Previous observations suggest that there are activated $\mathrm{T}$ cells in the uteri of women with anti-thyroid antibodies which may secrete cytokines that hamper successful pregnancy. These adverse effects can be mediated directly through $\mathrm{T}$ cells or indirectly through other cells, such as natural killer cells. ${ }^{39}$ 2) The presence of TA, which may act as an infertility factor and may delay conception increasing the rate of aging related miscarriage..$^{40}$ There are no clear recommendations as to whether euthyroid women should be tested for TA, but it seems that there is no benefit in treating these women with thyroid replacement therapy. ${ }^{41} \mathrm{Se}$ lenium is a trace element which is essential in thyroid hormone synthesis. Selenium substitution decreased TPO antibody levels in euthyroid subjects and women with recurrent pregnancy loss had lower selenium levels in their hair than controls. ${ }^{42}$ However, there is no RCT regarding the role of selenium substitution in women with RPL.

Further studies are required to determine whether all women with positive thyroidautoantibodies should not be started on thyroxin therapy during their pregnancies to decrease the miscarriage rate.

\section{DIABETES MELLITUS}

Pregestational diabetes, including type 1 and type 2 diabetes as well as other rare types of diabetes, complicates from $0.5 \%$ to $1 \%$ of all pregnancies. ${ }^{43}$ Studies show that patients suffering from this clinical condition run a significantly increased risk of spontaneous abortion, preterm labour, hypertensive disorders and operative deliveries. ${ }^{44,45}$ The main underlying cause is lethal embryonic malformations, the prevalence of which is increased in the case of poorly controlled diabetes during the periconceptional period. ${ }^{46,47,48}$ Glucose is teratogenic at high levels, and rates of congenital foetal anomalies are directly related to glycaemic control in the first trimester. Current evidence shows that well-controlled diabetes is not a risk factor for RPL and attention should first be given to optimal metabolic control of diabetic women during the preconceptional period. ${ }^{49,50}$

\section{POLYCYSTIC OVARY SYNDROME}

It has been estimated that $40 \%$ of pregnancies in women with PCOS will result in spontaneous loss. ${ }^{51}$ However, diagnostic criteria for this heterogeneous disorder have not been uniform, resulting in a wide range of reported prevalence of both miscarriage and RPL in PCOS patients. ${ }^{52}$ The majority of these studies have used polycystic ovary morphology alone to define PCOS and the results are extremely variable due to a variety of diagnostic and selection criteria employed. Polycistic ovarian morphology (PCO) per se is not a predictive of pregnancy loss in women with PRL. ${ }^{53}$ However, patients with PCOS may have several underlying contributing and interrelated factors, which have been reported in women with RPL, regardless of whether they have PCOS. These include obesity, hyperinsulinaemia, insulin resistance, hyperhomocysteinaemia, high levels of plasminogen activator inhibitor-1 factor, hyperandrogenaemia and poor endometrial receptivity. ${ }^{54,55}$ It is thought that obesity acts on female reproductive function through hyperinsulinaemia and, consequently, through its effect on androgen production. Some authors have argued that insulin resistance is a key factor in explaining the association between obesity, PCOS and recurrent miscarriages. ${ }^{56}$ Moreover, evidence has shown a possible association between insulin resistance, hyperhomocysteinaemia and the risk of PCOS. ${ }^{57}$ Recent studies have highlighted the presence of hypofibronolysis associated with high levels of PAI-1 as being a potential cause of RPL in women with PCOS. ${ }^{58}$ The effects of elevated PAI-1 may also be increased by elevated homocysteine, eventually leading to thrombosis. Moreover, plasma PAI-1 levels are associated with dyslipidaemia, hyperinsulinaemia and hypertension, three factors that contribute to the establishment of hyperhomocysteinaemia. ${ }^{59}$ Hence, PCOS involves several confounding factors that may contribute, individually or in a combination, to thrombosis and, eventually, may lead to RPL.

Metformin treatment of PCOS patients decreases insulin resistance, thus improving ovulation cycles and, therefore, conception rates in infertile women. ${ }^{60}$ Metformin has shown benefit in reducing the risk of miscarriage in women with a history of RPL and an abnormal glucose tolerance test result, ${ }^{61}$ but it is uncertain whether it decreases the rate of miscar- 
riage in PCOS patients as no proper RCT has been conducted.

\section{HYPERANDROGENISM}

Hyperinsulinaemia and hyperandrogenaemia are closely associated. Elevated androgens have detrimental effects on endometrial development and decrease oocyte and embryo viability, or they may have an indirect effect via the insulin pathways, perhaps via the insulin-like growth factor. ${ }^{62}$ The presence of an independent link between hyperandrogenaemia and RPL remains contentious. Several studies have investigated the androgen levels of women with RPL with conflicting results as to whether or not an association exists. In the past, two studies have shown that androgen levels in the follicular phase are higher in women dealing with RPL than in normal fertile controls. ${ }^{63}$ The apparent controversy is mainly attributed to the considerable variation in the specific androgens measured and to the phase of the menstrual cycle in which the measure was applied. The free testosterone and free androgen index (FAI) are considered to be the most sensitive methods and the assessment should be applied in the early follicular phase. ${ }^{64}$ The most recent large-scale study with measurement of the FAI in the early follicular phase demonstrated a significantly increased risk of miscarriage with increasing FAI (FAI >5). ${ }^{65}$ The prevalence of an elevated FAI was found to be $11 \%$ in the group of patients with RPL. Thus, hyperandrogenaemia seems to contribute to the pathology of RPL, but further studies should be conducted in order to determine whether therapeutic intervention to reduce the FAI improves the outcome in this group of women.

\section{REDUCED OVARIAN RESERVE}

Women with altered ovarian reserve markers, such as low AMH levels and a lower number of antral follicles (AFC) counted by transvaginal utrasonography (TVUS), usually experience a poor response to ovarian stimulation for assisted reproductive technology (ART). Whether the reduction in the primordial follicular pool is associated with an alteration in oocyte quality is a debated matter. Diminished ovarian reserve (DOR), defined as altered ovarian reserve markers with regular menstrual cycles, can be seen in the general population of young women conceiving naturally and is not necessarily considered a pathological entity. ${ }^{66}$ Diminished ovarian reserve can also result from a partial destruction of the primordial follicular pool due to surgical interventions on the ovaries (oophorectomy, cystectomy), to chemotherapies, medical conditions such as autoimmune oophoritis or to genetic factors such as permutations in the FMRI gene. Moreover, ovarian aging leads to a decrease in the ovarian reserve associated with an increase in foetal aneuploidy and pregnancy losses, which renders the study of a direct relationship between DOR and pregnancy loss difficult. Several publications however report an increased first trimester miscarriage rate in women with DOR. ${ }^{67-69}$ The evidence from the current medical literature is questionable for several reasons: 1) most studies are carried out in an infertile population, 2) the study sizes are small, 3) none of studies differentiates between DOR of different origins. Further studies in a non-infertile population with a characterization of the aetiology of DOR should be conducted before concluding on the association between DOR and pregnancy loss.

\section{CONCLUSIONS}

The definition, diagnosis and treatment of patients with a history of RPL remains difficult. The majority of sporadic losses before the $10^{\text {th }}$ week of gestation result from random numeric chromosome errors. However, endocrine disorders play an important role in RPL, especially in the early stages of gestation. Large, well-defined clinical trials focused on appropriate testing and treatment of endocrine disorders in RPL are essential to provide additional data on this challenging clinical condition.

\section{CONFLICT OF INTEREST}

None.

\section{REFERENCES}

1. Smith ML, Schust DJ, 2011 Endocrinology and recurrent early pregnancy loss. Semin Reprod Med 29: 482-490.

2. Jacobs PA, Hassold T 1987 Chromosome abnormalities: origin and etiology in abortions and livebirths. In: Vogel F, Sperling K, (eds) Human Genetics. Berlin: Springer-Verlag; pp, 233-244. 
3. Practice Committee of the American Society for Reproductive Medicine, 2012 Evaluation and treatment of recurrent pregnancy loss: a committee opinion. Fertil Steril 98: 1103-1111.

4. Mills JL, Simpson JL, Driscoll SG, et al, 1988 Incidence of spontaneous abortion among normal women and insulin-dependent women whose pregnancies were identified within 21 days of conception. N Engl J Med 319: 1617-1623.

5. Goddijn M, Joosten JH, Knegt AC, et al, 2004 Clinical relevance of diagnosing structural chromosome abnormalities in couples with repeated miscarriage. Hum Reprod 19: 1013-1017.

6. Kalinka J, Szekeres-Bartho J, 2005 The impact of dydrogesterone supplementation on hormonal profile and progesterone-induced blocking factor concentrations in women with threatened abortion. Am J Reprod Immunol 53: 166-171.

7. Szekeres-Bartho J, Balasch J, 2008 Progestagen therapy for recurrent miscarriage. Hum Reprod Update 14: 27 35.

8. Porter TF, Scott JR, 2005 Evidence-based care of recurrent miscarriage. Best Pract Res Clin Obstet Gynaecol 19: 85-101.

9. Arredondo F, Noble LS, 2006 Endocrinology of recurrent pregnancy loss. Semin Reprod Med 24: 33-39.

10. Insler V, 1992 Corpus luteum defects. Curr Opin Obstet Gynecol 4: 203-211.

11. Hensleigh PA, Fainstat T, 1979 Corpus luteum dysfunction: serum progesterone levels in the diagnosis and assessment of therapy for recurrent and threatened abortion. Fertil Steril 32: 396-400.

12. Cumming DC, Honore LH, Scott JZ, 1985 The late luteal phase in infertile women: comparison of simultaneous endometrial biopsy and progesterone levels. Fertil Steril 43: 715-719.

13. Arck PC, Rücke M, Rose M, et al, 2008 Early risk factors for miscarriage: a prospective cohort study in pregnant women. Reprod Biomed Online 17: 101-113.

14. Van der Gaast MH, Beier-Hellwig K, Fauser BC, et al, 2003 Endometrial secretion aspiration prior to embryo transfer does not reduce implantation rates. Reprod Biomed Online 7: 105-109.

15. Altmäe S, Esteban FJ, Stavreus-Evers A, et al, 2014 Guidelines for the design, analysis and interpretation of 'omics' data: focus on human endometrium., Hum Reprod Update 20: 12-28.

16. Karamardian LM, Grimes DA, 1992 Luteal phase deficiency effect of treatment on pregnancy rares. Am J Obstet Gynecol 167: 1391-1398.

17. Daya S, 1989 Efficacy of progesterone support for pregnancy in women with recurrent miscarriage: a meta-analysis of controlled trials. Br J Obstet Gynaecol 96: 275-280.

18. Oates-Whitehead RM, Haas DM, Carrier JA, 2003 Progestogen for preventing miscarriage. Cochrane
Database Syst Rev 4: CD003511.

19. Fatemi HM, Popovic-Todorovic B, Papanikolaou E, et al, 2007 An update of luteal phase support in stimulated IVF cycles. Hum Reprod Update 13: 581-590.

20. Fauser BC, Devroey P, 2003 Reproductive biology and IVF: ovarian stimulation and luteal phase consequences. Trends Endocrinol Metab 14: 236-242.

21. Boomsma CM, Kavelaars A, Eijkemans MJ , et al, 2010 Ovarian stimulation for in vitro fertilization alters the intrauterine cytokine, chemokine, and growth factor milieu encountered by the embryo. Fertil Steril 94: 1764-1768.

22. Frasor J, Park K, Byers M, et al, 2001 Differential roles for signal transducers and activators of transcription $5 \mathrm{a}$ and $5 \mathrm{~b}$ in PRL stimulation of ERalpha and ERbeta transcription . Mol Endocrinol 15: 2172-2181.

23. McNatty KP, Sawers RS, 1975 Relationship between the endocrine environment within the graafian follicle and the subsequent secretion of progesterone by human granulosa cells in culture. J Endocrinol 66: 391-400.

24. Hirahara F, Andoh N, Sawai K, Hirabuki T, Uemura T, Minaguchi H, 1998 Hypecprolacrinemic recurrent miscarriage and results of randomized bromocriptine treatment trials. Fertil Steril 70: 246-252.

25. Li TC, Spuijbroek MDEH, Tuckerman E, Anstie B, Loxley M, Laird SM, 2000 Endocrinological and endometrial factors in recurrent miscarriage. $\mathrm{Br} \mathrm{J}$ Obstet Gynaecol 107: 1975-1980.

26. Glinoer D, 1998 Thyroid hyperfunction during pregnancy. Thyroid 8: 859-864.

27. Millar LK, Wing DA, Leung AS, Koonings PP, Montoro MN, Mestman JH, 1994 Low birth weight and preeclampsia in pregnancies complicated by hyperthyroidism. Obstet Gynecol ; 84: 946-949.

28. Anselmo J, Cao D, Karrison T, Weiss RE, Refetoff S, 2004 Fetal loss associated with excess thyroid hormone exposure. JAMA 292: 691-695.

29. Allan WC, Haddow JE, Palomaki GE, et al, 2000 Maternal thyroid deficiency and pregnancy complications: implications for population screening. J Med Screen 7: 127-130.

30. Abalovich M, Gutierrez S, Alcaraz G, Maccallini G, Garcia A, Levalle O, 2002 Overt and subclinical hypothyroidism complicating pregnancy. Thyroid 12: 63-68.

31. Wakim AN, Polizotto SL, Buffo MJ, Marrero MA, Burholt DR, 1993 Thyroid hormones in human follicular fluid and thyroid hormone receptors in human granulosa cells. Fertil Steril 59: 1187-1190.

32. Clifford K, Rai R, Watson H, Regan L, 1994 An informative protocol for the investigation of recurrent miscarriage: preliminary experience of 500 consecutive cases. Hum Reprod 9: 1328-1332.

33. Baloch Z, Carayon P, Conte-Devolx, et al, 2003 Guidelines Committee, National Academy of Clinical Biochemistry. Laboratory medicine practice. guidelines. Laboratory support: for the diagnosis and monitoring 
of thyroid disease. Thyroid 13: 3-126.

34. Poppe K, Velkeniers B, Glinoer D, 2007 Thyroid disease and female reproduction. Clin Endocrinol 66: 309-321.

35. Marai I, Carp H, Shai S, Shabo R, Fishman G, Shoenfeld Y, 2004 Autoantibody Panel Screening in Recurrent Miscarriages. Am J Reprod Immunol 51: 235-240.

36. Stagnaro-Green A, Roman SH, Cobin RH, el-Harazy E, Alvarez-Marfany M, Davies TF, 1990 Detection of at-risk pregnancy by means of highly sensitive assays for thyroid autoantibodies. JAMA 264: 1422-1425.

37. Roberts J, Jenkins C, Wilson R, et al, 1996 Recurrent miscarriage is associated with increased numbers of CD5/20 positive lymphocytes and an increased incidence of thyroid antibodies. Eur J Endocrinol 134: 84-86.

38. Twig G, Shina A, Amital H, Shoenfeld Y, 2012 Pathogenesis of infertility and recurrent pregnancy loss in thyroid autoimmunity. J Autoimmun 38: J275-281.

39. Konova E, 2010 The role of NK cells in the autoimmune thyroid disease-associated pregnancy loss. Clin Rev Allergy Immunol 39: 176-184.

40. Lejeune B, Grun JP, de Nayer P, Servais G, Glinoer D, 1993 Antithyroid antibodies underlying thyroid abnormalities and miscarriage or pregnancy induced hypertension. Br J Obstet Gynaecol 100: 669-672.

41. Rushworth FH, Backos M, Rai R, Chilcott IT, Baxter N, Regan L, 2000 Prospective pregnancy outcome in untreated recurrent miscarriers with thyroid autoantibodies. Hum Reprod 15: 1637-1639.

42. Prummel MF, Wiersinga WM, 2004 Thyroid autoimmunity and miscarriage. Eur J Endocrinol 150: 751-755.

43. Gabbe SG, Graves CR, 2003Management of diabetes mellitus complicating pregnancy. Obstet Gynecol 102: 857-868.

44. Sibai BM, 2000 Risk factors, pregnancy complications, and prevention of hypertensive disorders in women with pregravid diabetes mellitus. J Matern Fetal Med 9: 62-65.

45. Melamed N, Hod M, 2009 Perinatal mortality in pregestational diabetes. Int J Gynaecol Obstet 104: Suppl 1: 20-24.

46. Lucas MJ, Leveno KJ, Williams ML, Raskin P, Whalley PJ, 1989 Early pregnancy glycosylated hemoglobin, severity of diabetes, and fetal malformations. Am J Obstet Gynecol 16: 426-431.

47. Kitzmiller JL, Wallerstein R, Correa A, Kwan S, 2010 Preconception care for women with diabetes and prevention of major congenital malformations. Birth Defects Res A Clin Mol Teratol 88: 791-803.

48. Gutaj P, Zawiejska A, Wender-Ożegowska E, Brązert J, 2013 Maternal factors predictive of first-trimester pregnancy loss in women with pregestational diabetes. Pol Arch Med Wewn 123: 21-28.

49. Rai R, Regan L, 2006 Recurrent miscarriage. Lancet 368: 601-611.

50. Nicholson W, Baptiste-Roberts K, 2011 Oral hypoglycaemic agents during pregnancy: The evidence for effectiveness and safety. Best Pract Res Clin Obstet Gynaecol 25: 51-63.

51. Rai R, Backos M, Rushworth F, Regan L, 2000 Polycystic ovaries and recurrent miscarriage--a reappraisal. Hum Reprod 15: 612-615.

52. Rotterdam ESHRE/ASRM-Sponsored PCOS Consensus Workshop Group, 2004 Revised 2003 consensus diagnostic criteria and long-term health risks related to polycysic ovary syndrome. Fertil Steril 81: 19-25.

53. Christiansen OB, 2006 Evidence-based investigations and treatments of recurrent pregnancy loss. Curr Opin Obstet Gynecol 18: 304-312.

54. Wang JX, Davies MJ, Norman RJ, 2002 Obesity increases the risk of spontaneous abortion during infertility treatment. Obes Res 10: 551-554.

55. Angioni S, Sanna S, Magnini R, Melis GB, Fulghesu AM, 2011 The quantitative insulin sensitivity check index is not able to detect early metabolic alterations in young patients with polycystic ovarian syndrome. Gynecol Endocrinol 27: 468-474.

56. Tian L, Shen H, Lu Q, Norman RJ, Wang J, 2007 Insulin resistance increases the risk of spontaneous abortion after assisted reproduction technology treatment. Clin Endocrinol Metab 92: 1430-1433.

57. Schachter M, Raziel A, Friedler S, Strassburger D, Bern O, Ron-El R, 2003 Insulin resistance in patients with polycystic ovary syndrome is associated with elevated plasma homocysteine. Hum Reprod 18: 721-727.

58. Sun L, Lv H, Wei W, Zhang D, Guan Y, 2010 Angiotensin-converting enzyme D/I and plasminogen activator inhibitor-1 4G/5G gene polymorphisms are associated with increased risk of spontaneous abortions in polycystic ovarian syndrome. J Endocrinol Invest 33: 77-82.

59. Bastard JP, Piéroni L, Hainque B, 2000 Relationship between plasma plasminogen activator inhibitor 1 and insulin resistanceDiabetes Metab Res Rev 16: 192-201.

60. Tang T, Lord JM, Norman RJ, Yasmin E, Balen AH, 2012 Insulin-sensitising drugs (metformin, rosiglitazone, pioglitazone, D-chiro-inositol) for women with polycystic ovary syndrome, oligo amenorrhoea and subfertility. Cochrane Database Syst Rev 5: CD003053.

61. Zolghadri J, Tavana Z, Kazerooni T, et al, 2008 Relationship between abnormal glucose tolerance test and history of previous recurrent miscarriages, and beneficial effects of metformin in these patients: a prospective clinical study. Fertil Steril 90: 727-730.

62. Okon MA, Laird SM, Tuckerman E, Li TC, 1998 Serum androgen levels in women who suffer recurrent miscarriage and their correlation with markers of endometrial function. Fertil Steril 69: 682-690.

63. Bussen S, Sutterlin M, Steck T, 1999 Endocrine abnormalities during the follicular phase in women with recurrent spontaneous abortions. Hum Reprod 14:18-20.

64. Rotterdam ESHRE/ASRM-Sponsored PCOS consensus workshop group, 2004 Revised 2003 consensus on diagnostic criteria and long-term health risks related 
to polycystic ovary syndrome PCOS. Hum Reprod 19: 41-47.

65. Cocksedge KA, Saravelos SH, Wang Q, Tuckerman E, Laird SM, Li TC, 2008 Does free androgen index predict subsequent pregnancy outcome in women with recurrent miscarriage? Hum Reprod 23: 797-802.

66. Massé V, Ferrari P, Boucoiran I , et al, 2011 Normal serum concentrations of anti-Mullerian hormone in a population of fertile women in their first trimester of pregnancy. Hum Reprod 26: 3431-3436.
67. Trout SW, Seifer DB, 2000 Do women with unexplained recurrent pregnancy loss have higher day 3 serum FSH and estradiol values? Fertil Steril 74: 335-337.

68. Levi AJ, Raynault MF, Bergh PA, et al, 2001 Reproductive outcome in patients with diminished ovarian reserve. Fertil Steril 76: 666-669.

69. Elter K, Kavak ZN, Gokaslan H, et al, 2005 Antral follicle assessment after down-regulation may be a useful tool for predicting pregnancy loss in in vitro fertilization pregnancies. Gynecol Endocrinol 21: 33-37. 